\title{
THE DIFFICULTIES AND PITFALLS OF TRANSPLANTA- TION OF THE URETERS INTO THE LARGE BOWEL
}

\section{R. C. Coffey}

$\mathrm{P}^{\mathrm{i}}$ ROBABLY no more important nor bewildering problem has ever engaged the attention of clinical and experimental surgeons than that of transplanting the ureters into the large bowel. In no other place in the human body does a single principle and the technical procedure necessary to make the principle effective, control such a large field of surgery. For, upon this single procedure, aII surgery contemplating the elimination of the bladder as a reservoir for urine depends.

As one reads the literature and records of the attempts made to solve this problem, the most surprising thing encountered is that while the statement to the effect that the ureter opens into the bladder "by a constricted orifice after having passed obliquely for nearly an inch between its muscular and mucous coats" has been in Gray's anatomy for more than thirty years, its full significance was not generally appreciated by experimenters. The knowledge that the ureter ran beneath the loose mucosa for nearly an inch before entering the bladder apparently made little impression for the reason that the problem involved had not been isolated and defined. The problem itself was discovered by accident in a set of experiments in which it was necessary to transplant the bile duct into the intestine below its normal site. Two weeks later, it was invariably found that the duct which had been transplanted directly into the intestine was enormously dilated. This was observed in every case. Obviously a fundamental principle was involved. By tracing bile ducts along their courses into the intestines of dogs, it was found that the duct always ran beneath the loose mucous membrane for a distance before entering the Iumen of the bowel. Ducts surgically placed in this submucous position were found not dilated two weeks after transplantation. The problem had been isolated and its solution had been found. The abstract problem was the delivery of fluids from a viscus of low pressure to one of higher pressure. The solution was found in the production of valve action. The immediate problem at hand was the delivery of the fluid of a secreting organ in the ducts of which the pressure must be low and regular, into a second hollow viscus in which the pressure is high and irregular. The solution was the production of a valve from the nonmotile mucous membrane of the second viscus.

Considered broadly, it would seem that the valve is the only mechanism by which fluids and gases, at a given temperature, may be transmitted from a chamber of low pressure to one of higher pressure. Working upon this principle, it was possible to present frve undilated ureters and undamaged kidneys of dogs operated upon 60 to 167 days prior to the removal of the specimens, to the Surgical Section of the American Medical Association at St. Louis in June, 19 Io. Dr. Charles H. Mayo, who was Chairman of the Section at that time, soon applied the principle and technique to the human being. Since that time he has operated upon nearly a hundred patients. His results, taken with those of Lower and operators of less experience, have furnished indisputable proof that ureters thus transplanted safely deliver the urine into the large bowel with very little danger of infection of the kidney as a late result. But the fact that even in these most skilled and experienced hands the immediate mortality from infection was considerable while in less skilled hands it was almost prohibitive, caused many urologists to condemn the procedure as unjustifiable. Even in the hands of these most skilled operators, the fact that only one ureter could be transplanted at a time, thus requiring two or three major operations in removing the bladder, made the operation impractical for most conditions other than exstrophy of the bladder. 
The development of the bilateral tube technique described in the November, 1928 Surgery, Gynecology and Obstetrics has placed the operation on an entirely different plane and makes it available for any condition in which it is necessary to dispense with the bladder as a urinary reservoir. For it must be conceded that the successful operation upon thirteen consecutive patients by the same operator without operative death or demonstrable injury to the kidneys, aside from a single fistula from one ureter, entirely removes the element of chance as an importantfactor. Yet it is easy to foresee many difficulties in the way of establishing this operation as a generally adopted procedure.

One of the greatest difficulties in the way is the tendency of most surgeons to modify a new operative procedure so as to harmonize it with their particular principles. Second, it is going to be difficult to convince many surgeons of the importance of the quarantine which in reality is the means of making the operation extra-peritoneal in effect. There is no gainsaying the fact that this is not a simple operation. It is doubtful if there is any abdominal surgery in which a meticulously perfect technique is more important and in which there are so many details to be looked after. A long and arduous experience, both experimental and clinical, forces the conclusion that there is no important detail described in this article which may be safely omitted. I believe that the quarantine will prove to be an absolute essential if one is to have uniformly good success.

The greatest obstacle of all, however, to the universal adoption of this operation, is that it is essentially a highly technical intestinal operation. It is primarily not a urological operation. The great majority of the cases are diagnosed by urologists, many of whom have had very little experience in intestinal surgery and who are therefore likely to meet with frequent disaster.

How are these inconsistencies to be reconciled? A few urologists are also skilled intestinal surgeons. No doubt, still other enterprizing urologists will by cadaver practice, experimental surgery on animals and a large clinical experience, master this particular feature of intestinal surgery but it is more than likely that the majority of urologists will not. What will this latter group do with their cases? Will they cooperate with the general surgeon who is skilled in intestinal surgery or with urologists who have mastered this technique, or will they, basing their opinions on unfortunate personal experiences, condemn the operation as unjustifiable? 